\title{
High-concentration glucose enhances invasion in invasive ductal breast carcinoma by promoting Glut1/MMP2/MMP9 axis expression
}

\author{
XIAN-FU SUN ${ }^{1}$, YING-BO SHAO ${ }^{1}$, MING-GE LIU ${ }^{2}$, QI CHEN ${ }^{1}$, \\ ZHAO-JUN LIU ${ }^{1}$, BIN XU ${ }^{1}$, SU-XIA LUO ${ }^{3}$ and HUI LIU ${ }^{1}$ \\ Departments of ${ }^{1}$ Galactophore, ${ }^{2}$ Pathology and ${ }^{3}$ Medical Oncology, \\ Henan Cancer Hospital, Zhengzhou, Henan 450008, P.R. China
}

Received July 8, 2015; Accepted October 13, 2016

DOI: $10.3892 / \mathrm{ol} .2017 .5843$

\begin{abstract}
Type 2 diabetes mellitus (T2DM) has been considered to be a risk factor for numerous human cancers. Hyperglycemia is one of the most direct internal environmental changes for patients with T2DM. Increasing evidence reveals that a high concentration of glucose can promote tumor progression, while its role for migration and invasion of invasive ductal breast carcinoma (IDBC) cells remains unclear. In the present study, it was demonstrated that IDBC patients with T2DM suffered an increased tumor size and more frequent lymphatic and distant metastasis compared with those without T2DM $(\mathrm{P}<0.05)$. MCF-7 breast carcinoma cells, which were cultured in a high glucose concentration medium (25.00 $\mathrm{mM})$, exhibited increased invasion $(\mathrm{P}<0.05)$. In addition, the expression of glucose transporters (Gluts), matrix metalloproteinase 2 (MMP2) and matrix metalloproteinase 9 (MMP9) in IDBC tissues with T2DM was significantly higher compared to those without T2DM. Downregulation of glucose transporter 1 (Glut1) by small interfering RNA may markedly suppress MCF-7 cell invasion as well as the expression of MMP2 and MMP9. These results suggest that T2DM can affect the malignant features of tumors in IDBC. The high glucose concentration in the tumor microenvironment may enhance IDBC invasion via upregulating Glut1/MMP2/MMP9 axis expression.
\end{abstract}

\section{Introduction}

Breast cancer is one of the most common types of female cancer (1). The genesis of breast cancer is concealed, its progress is rapid and the mortality rate is high. With the development

Correspondence to: Dr Hui Liu, Department of Galactophore, Henan Cancer Hospital, No. 127 Dongming Road, Zhengzhou, Henan 450008, P.R. China

E-mail: hchliuhui@126.com

Key words: type 2 diabetes mellitus, invasive ductal breast carcinoma, Glut1, MMP2, MMP9, invasion of living standard, the morbidity of type 2 diabetes mellitus (T2DM) increases rapidly (2). At present, numerous studies have found that T2DM is a systemic disease, and associated with the progression of several human malignant tumors $(3,4)$.

Ma et al (5) analyzed the prognosis of 865 early triple-negative breast cancer (TNBC) patients and demonstrated that patients with TNBC and T2DM had a shorter disease free survival (DFS) time and more frequent distant metastasis. Tumor microenvironments, consisting of numerous stromal cells, cytokines and chemokines, markedly enhance tumor growth, invasion and metastasis (6). Adham et al (7) demonstrated that the glucose concentration of the medium regulates tumor cell epithelial mesenchymal transition (EMT). Therefore, the present study inferred that high-concentration glucose in the tumor microenvironment may promote invasion of breast cancer cells.

Cancer cells generate adenosine triphosphate mainly by the process of anaerobic glycolysis, which is termed the Warburg effect $(8,9)$. However, due to the hydrophobicity of glucose molecules, the process of glucose passing through cell lipid bilayers must rely on the assistance of a glucose transporter (Glut) (10). Therefore, the expression and functions of Gluts partly determine the degree of the Warburg effect. Glucose transporter 1 (Glut1), encoded by $S L C 2 A 1$, is a member of the major facilitator superfamily. Due to the high affinity of Glut1 for glucose molecules, it can transport glucose molecules into cells even at a low concentration. Studies have found that Glut1 is overexpressed in the retina and glomerulus of T2DM patients $(11,12)$. As anaerobic glycolysis is an inefficient method of energy production, cancer cells require enhanced glucose supply. Ectopic expression levels of Glut1 have been observed in several types of cancer, including endometrial (13), non-small lung (14) and colorectal (15). These studies identified Glut1 as an important prognostic indicator for tumorigenesis. However, whether hyperglycemia caused by T2DM in the tumor microenvironment can upregulate the expression of Glut1 and enhance the invasion ability in breast cancer cells remains unclear.

In the present study, it was demonstrated that the expression of Glut1 was higher in breast cancer tissues with T2DM than without T2DM. High glucose concentration may increase the 
expression of Glut1, and consequently enhance cell invasion by upregulating the expression of matrix metalloproteinase 2 (MMP2) and matrix metalloproteinase 9 (MMP9) in vitro.

\section{Materials and methods}

Patients and samples. A total of 120 patients, including 60 patients with T2DM and 60 patients without T2DM, were enrolled in the present study and underwent surgery between April 2011 and June 2014. The age range was between 29 and 69 years, and the median age was 51 years. All resected tumor tissues were identified as invasive ductal breast carcinoma (IDBC) by senior pathologists. Patients did not receive chemotherapy and/or radiotherapy prior to surgery. Clinical data was collected from medical records. The IDBC tissues and tumor adjacent tissues $(>2 \mathrm{~cm}$ distance from the surgical boundary) were stored in $4 \%$ paraformaldehyde solution for immunohistochemistry (IHC) or liquid nitrogen for reverse transcription-quantitative polymerase chain reaction (RT-qPCR).

All protocols were approved by the Henan Cancer Hospital Ethics Committee (Henan, China). Informed consent was obtained and signed by each patient.

Cell culture. The MCF-7 breast cancer cells were obtained from the Institute of Biochemistry and Cell Biology, Chinese Academy of Sciences (Shanghai, China). Cells were maintained under different glucose concentrations, either $5.56 \mathrm{mM}$ or $25.00 \mathrm{mM}$, and cultured in a $37^{\circ} \mathrm{C}$ humidified incubator with $5 \% \mathrm{CO}_{2}$. The logarithmic growth phase cells were harvested for additional assays.

Transwell assay. The MCF-7 cells cultured in different glucose concentrations were suspended with reduced serum Dulbecco's modified Eagle's medium (DMEM; Invitrogen; Thermo Fisher Scientific, Inc., Waltham, MA, USA) and adjusted the density to $2.5 \times 10^{5} / \mathrm{ml}$. In total, $200 \mu \mathrm{l}$ cell suspension was added into the upper well of Matrigel-coated (BD Biosciences, Franklin Lakes, NJ, USA) $8-\mu \mathrm{m}$ pore Transwell inserts (Nalge Nunc International, Penfield, NY, USA), and $750 \mu 1$ DMEM with 10\% fetal bovine serum (Invitrogen; Thermo Fisher Scientific, Inc.) was added into the lower well. Cells were incubated in a $37^{\circ} \mathrm{C}$ humidified incubator with $5 \% \mathrm{CO}_{2}$ for $24 \mathrm{~h}$. Membranes were removed, and uninvaded cells were scraped off and stained with crystal violet. Cell numbers were counted using ImageJ software (National Institutes of Health, Bethesda, MD, USA). The experiments were performed in triplicate.

IHC staining. IHC was performed on paraformaldehyde-fixed paraffin sections. The SP link IHC Detection kit (Biotin-Streptavidin HRP Detection systems; catalog no. SP-9001) was purchased from OriGene Technologies, Inc. (Beijing, China). In brief, rabbit anti-human polyclonal Glut1 (catalog no. sc-7903; dilution, 1:100), rabbit anti-human polyclonal MMP2 (catalog no. sc-10736; dilution, 1:100) and rabbit anti-human polyclonal MMP9 (catalog no. sc-10737; dilution, 1:100) antibodies were purchased from Santa Cruz Biotechnology, Inc. (Dallas, TX, USA) and used to detect the protein expression in IDBC tissues. Tissue sections were incubated with primary antibodies at $4^{\circ} \mathrm{C}$ for $24 \mathrm{~h}$. Goat anti-rabbit secondary antibodies from the SP
Table I. Primer sequences

\begin{tabular}{ll}
\hline Gene & \multicolumn{1}{c}{ Primer sequence } \\
\hline Glut1 & \\
Sense & 5'-GTCTGGCATCAACGCTGTCT-3' \\
Antisense & 5'-ACCACACAGTTGCTCCACATAC-3' \\
MMP2 & \\
Sense & 5'-AAGGATGGCAAGTAC GGCTT-3' \\
Antisense & 5'-CGCTGGTACAGCTCTCATACTT-3' \\
MMP9 & \\
Sense & 5'-CCTGGAGACCTGAGAACCAATC-3' \\
Antisense & 5'-CACCCGAGTGTAACCATAGC-3' \\
$\beta$-actin & \\
Sense & 5'-CTCCATCCTGGCCTCGCTGT-3' \\
Antisense & 5'-GCTGTCACCTTCACCGTTCC-3' \\
\hline
\end{tabular}

Glut1, glucose transporter 1; MMP2, matrix metalloproteinase 2; MMP9, matrix metalloproteinase 9.

link IHC Detection kit were added to the sections and tissue sections were incubated at $37^{\circ} \mathrm{C}$ for $1 \mathrm{~h}$. Protein expression was visualized using 3,3-diaminobenzidine tetrahydrochloride (OriGene Technologies, Inc.) and observed through a BX46 upright microscope (Olympus Corporation, Tokyo, Japan). The staining results for the Glut1, MMP2 and MMP9 proteins were semi-quantitatively calculated by multiplying the staining intensity and the percentage of positive normal cells, as previously reported (16).

Small interfering RNA (siRNA) transfection. Glut1 specific siRNA (catalogue no. sc-35,493; Santa Cruz Biotechnology, Inc.) was transfected into MCF-7 cells by Lipofectamine ${ }^{\circledR} 2000$ (Invitrogen; Thermo Fisher Scientific, Inc.) according to the manufacturer's protocol. Scrambled siRNA (catalogue no. sc-37,007; Santa Cruz Biotechnology, Inc.) was used as a negative control. Reduced serum medium was changed into complete medium $6 \mathrm{~h}$ subsequent to transfection. Cells were harvested $48 \mathrm{~h}$ subsequent to transfection, and then used for additional experiments.

RT- $q P C R$. Total RNA was isolated from tissues and MCF-7 cells using TRIzol ${ }^{\circledR}$ reagent (Invitrogen; Thermo Fisher Scientific, Inc.) according to the manufacturer's protocol. A quantitative one-step Perfect Real Time RT-qPCR (SYBR-Green I) kit (Takara Biotechnology Co., Ltd., Dalian, China) was used to detect the expression of Glut1, MMP2 and MMP9, according to the manufacturer's protocol. The human $\beta$-actin gene was used as a reference gene. Primers (Table I) were synthesized by AuGCT DNA-Syn Biotechnology Co., Ltd. (Beijing, China). Relative mRNA expression was calculated using the $2^{-\Delta \Delta C q}$ method (17). All experiments were performed at least in triplicate.

Western blot analysis. Cells were cleaved with radioimmunoprecipitation assay (HEART Biotech, Xi'an, China) reagent on ice, and then the supernatant was obtained to determine 
Table II. Clinical features of IDBC patients with or without T2DM ( $n=120)$.

\begin{tabular}{|c|c|c|c|c|}
\hline Clinical features & IDBC with T2DM $(n=60)$ & IDBC without T2DM $(n=60)$ & $\chi^{2}$ & P-value \\
\hline Age, years & & & 2.155 & 0.142 \\
\hline$<60$ & 31 & 23 & & \\
\hline$\geq 60$ & 29 & 37 & & \\
\hline Menopause & & & 1.429 & 0.232 \\
\hline Yes & 39 & 45 & & \\
\hline No & 21 & 15 & & \\
\hline Tumor size, $\mathrm{cm}$ & & & 6.806 & $0.009^{\mathrm{a}}$ \\
\hline$\leq 2$ & 17 & 31 & & \\
\hline$>2$ & 43 & 29 & & \\
\hline Number of nodules & & & 1.269 & 0.260 \\
\hline 1 & 20 & 26 & & \\
\hline$\geq 2$ & 40 & 34 & & \\
\hline Histopathological grade & & & 0.839 & 0.360 \\
\hline $\mathrm{G} 1-\mathrm{G} 2$ & 25 & 30 & & \\
\hline G3 & 35 & 30 & & \\
\hline Lymphatic metastasis & & & 10.848 & $0.001^{\mathrm{a}}$ \\
\hline No & 19 & 37 & & \\
\hline Yes & 41 & 23 & & \\
\hline Distant metastasis & & & 5.711 & $0.017^{\mathrm{a}}$ \\
\hline No & 27 & 40 & & \\
\hline Yes & 33 & 20 & & \\
\hline
\end{tabular}

${ }^{\text {a }}<0$.05. IDBC, invasive ductal breast carcinoma; T2DM, type II diabetes mellitus.

protein contents via a bicinchoninic acid kit (EMD Millipore, Billerica, MA, USA), according to the manufacturer's protocol. Proteins were separated by vertical electrophoresis and transferred to a polyvinylidene fluoride membrane (EMD Millipore). Rabbit anti-human polyclonal Glut1 (dilution, 1:1,000), rabbit anti-human polyclonal MMP2 (dilution, 1:1,000), rabbit anti-human polyclonal MMP9 (dilution, $1: 1,000)$ and mouse anti-human monoclonal $\beta$-actin (catalog no. sc-47778; dilution, 1:5,000) antibodies were purchased from Santa Cruz Biotechnology, Inc. and used to detect the protein expression. Protein expression intensity was developed by the enhanced chemiluminescent reagent (EMD Millipore).

Statistical analysis. Measurement data are presented as the mean \pm standard deviation. SPSS (version 13.0; SPSS, Inc., Chicago, IL, USA) was used for the statistical tests, which consisted of the Pearson's $\chi^{2}$ test and a two-tailed Student's t-test. $\mathrm{P}<0.05$ was considered to indicate a statistically significant difference.

\section{Results}

Association between T2DM and the clinical features of IDBC patients. The present study analyzed the differences between clinical features in IDBC patients with or without T2DM by $\chi^{2}$ test. As shown in Table II, the results demonstrated that IDBC patients with T2DM more commonly had a larger tumor size ( $\geq 2 \mathrm{~cm} ; \mathrm{P}=0.009)$, lymphatic metastasis $(\mathrm{P}=0.001)$ and distant metastasis $(\mathrm{P}=0.017)$. The present study infers that patients with IDBC and T2DM exhibit increased metastasis, which indicates a reduced survival rate.

High glucose microenvironment enhances MCF-7 cell invasion. Hyperglycemia is one of the most direct changes in T2DM patients. In order to investigate the effect of hyperglycemia in tumor metastasis, the present study first detected the fasting blood glucose (FBG) level in IDBC patients with T2DM. Compared to non-metastatic IDBC (nmIDBC) patients, the FBG level was increased in metastatic IDBC (mIDBC) patients (7.32 \pm 0.62 vs. $10.79 \pm 1.28 \mathrm{mM} ; \mathrm{P}=0.0310$; Fig. $1 \mathrm{~A})$. In addition, Transwell assay demonstrated that MCF-7 cells cultured in high glucose concentration $(25 \mathrm{mM})$ medium acquired stronger invasion ability compared with those cultured in low glucose concentration $(5.56 \mathrm{mM})$ medium $(35.74 \pm 4.03$ vs. $19.48 \pm 3.12 ; \mathrm{P}=0.030$; Fig. $1 \mathrm{~B}$ and $\mathrm{C}$ ).

Expression of Glut1, MMP2 and MMP9 in IDBC tissues. In order to investigate the mechanisms of IDBC metastasis enhanced by T2DM, the present study detected the mRNA and protein expressions of Glut1, MMP2 and MMP9 in IDBC tissues. The mRNA and protein expression of Glut1, MMP2 and MMP9 in IDBC with T2DM tissues were significantly higher compared with IDBC without T2DM tissues (Fig. 2; $\mathrm{P}=0.02, \mathrm{P}=0.031, \mathrm{P}=0.010$, respectively). 

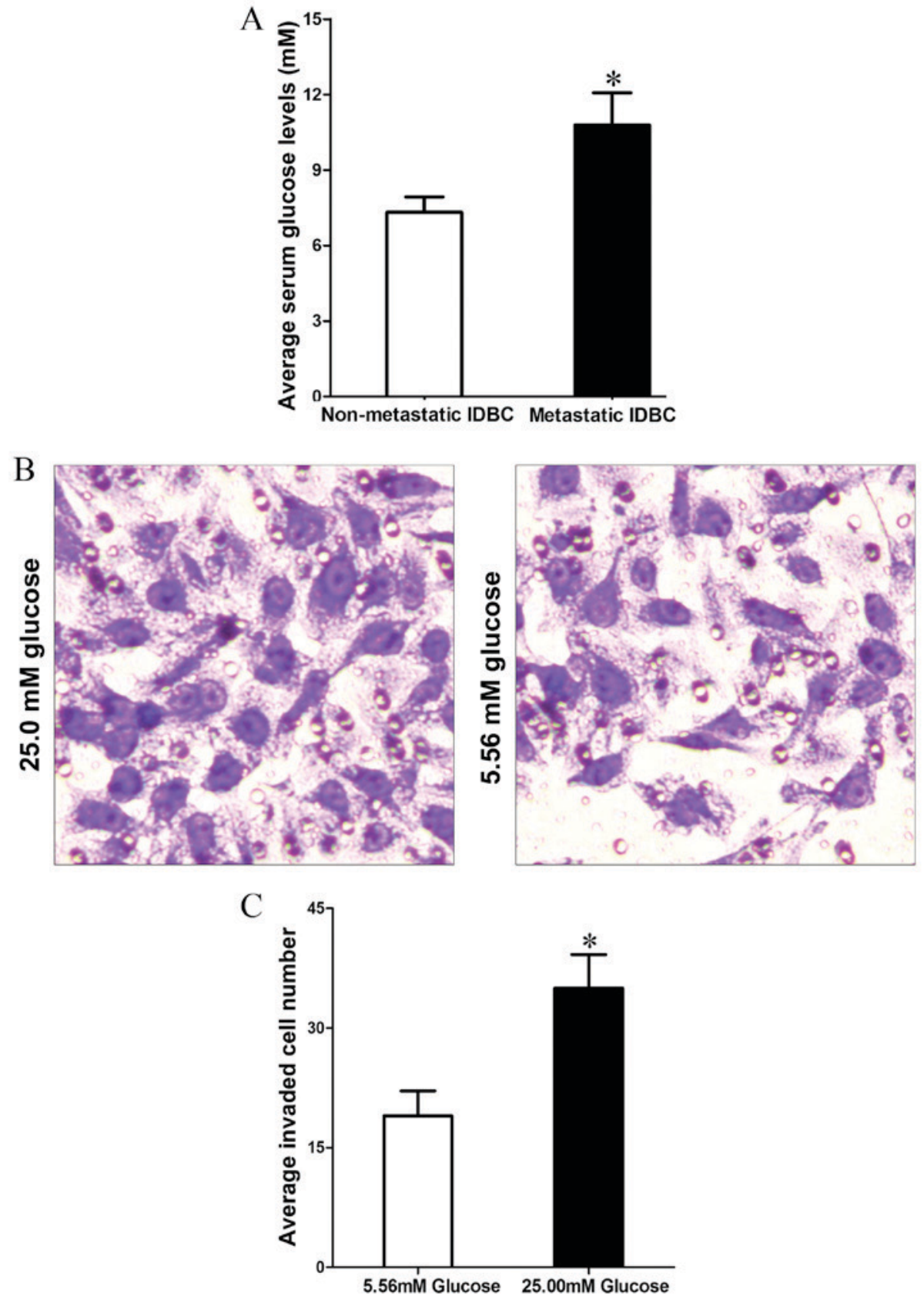

Figure 1. (A) Fasting blood glucose level was higher in patients with metastatic IDBC than in patients with non-metastatic IDBC. (B) Transwell assay results demonstrated that MCF-7 cells cultured in a high-concentration glucose medium (25.00 mM) increased invasive ability (magnification, x200), (C) Quantification of MCF-7 cell invasion. "P<0.05. IDBC, invasive ductal breast carcinoma.

Downregulating Glut1 inhibits MCF-7 cell invasion in a high glucose microenvironment. The present study transfected Glut1-specific siRNA into MCF-7 cells, which were cultured in high glucose $(25 \mathrm{mM})$ medium, and demonstrated that the transfection markedly inhibited Glut1 expression (Fig. 3A and B; $\mathrm{P}=0.033$ ). Transwell assay additionally verified that downregulation of Glut1 may suppress the invasion ability of MCF-7 cells cultured in high glucose medium (33.43 \pm 3.28 vs. 21.96 \pm 2.15 ; Fig. $3 \mathrm{C}$ and $\mathrm{D}$; $\mathrm{P}=0.027$ ).

Downregulating Glut1 inhibits MMP2 and MMP9 expression in high glucose microenvironment. MMPs, particularly MMP2 and MMP9, are crucial for cancer cell invasion, and their expression may be regulated by Glut1 (18). Therefore, the present study detected the expression of MMP2 and MMP9 by RT-qPCR and western blot analysis in MCF-7 cells subsequent to transfection. It can be observed in Fig. 4 that mRNA and protein expression of MMP2 and MMP9 are repressed subsequent to the downregulation of Glut1 $(\mathrm{P}=0.021, \mathrm{P}=0.034$, respectively).

\section{Discussion}

The incidence of breast cancer accounts for $20 \%$ of all female cancers $(19,20)$, and numerous patients present with one or more metabolic disease, such as T2DM. Studies have found that T2DM is an important risk factor for gastric cancer, endometrial cancer and numerous other cancer prognoses (21). 
A

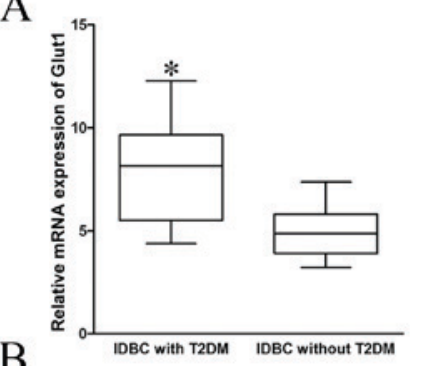

$\mathrm{B}$
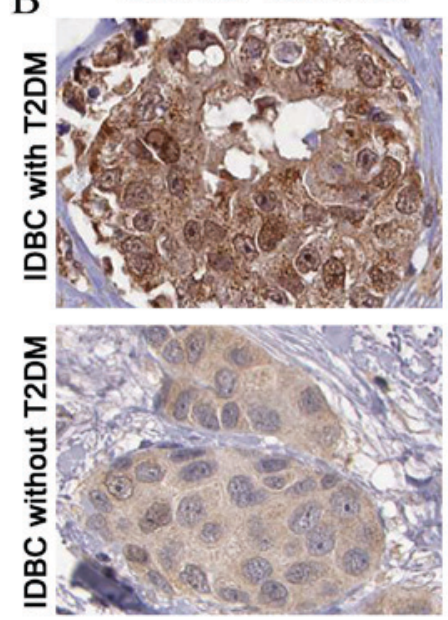

Glut1
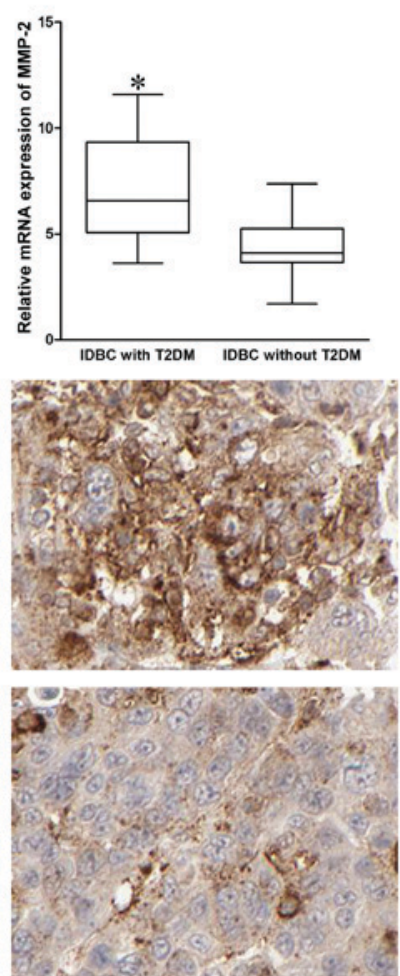

MMP2
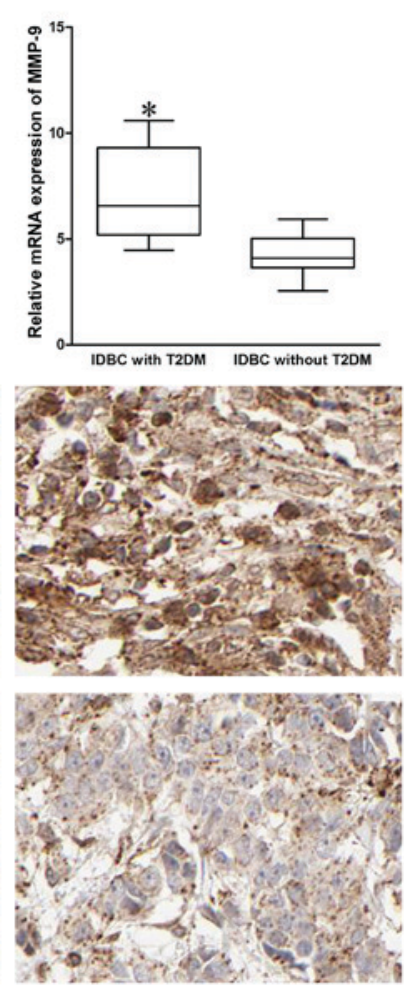

MMP9

Figure 2. (A) mRNA expression of Glut1, MMP2 and MMP9 was increased in IDBC patients with T2DM compared with patients without T2DM. (B) Immunohistochemical staining demonstrated that the protein expression of Glut1, MMP2 and MMP9 in IDBC patients with T2DM was highly upregulated (magnification, $\mathrm{x} 400$; DAB). ${ }^{*} \mathrm{P}<0.05$. IDBC, invasive ductal breast carcinoma; Glut1, glucose receptor 1; MMP2, matrix metalloproteinase 2; MMP9, matrix metalloproteinase 9; T2DM, type II diabetes mellitus.

A

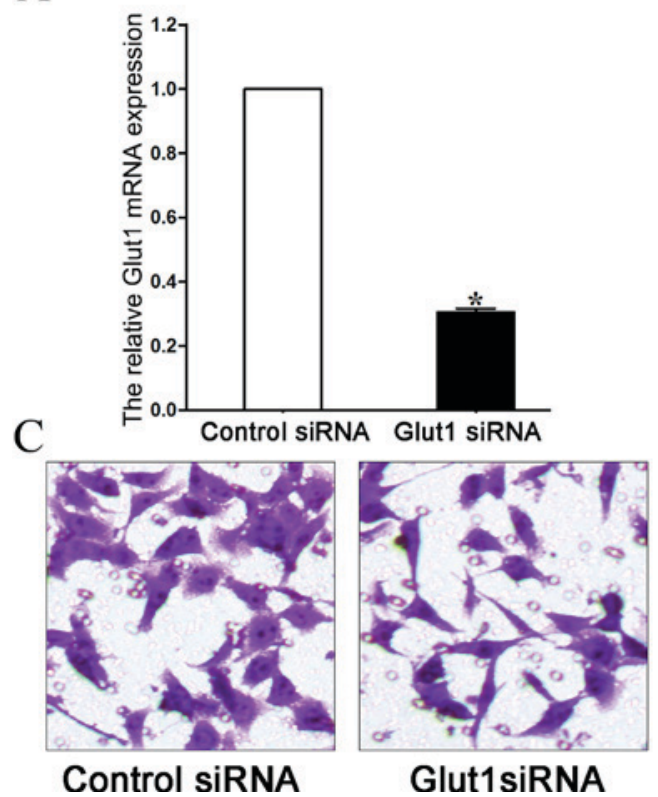

B

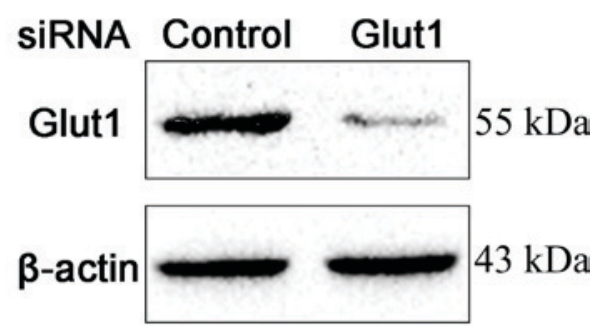

D



Figure 3. (A) siRNA inhibited Glut1 mRNA expression in MCF-7 cells. (B) siRNA inhibited Glut1 protein expression in MCF-7 cells. (C) Downregulation of Glut1 suppressed MCF-7 cell invasion (magnification, x200). (D) Quantification of MCF-7 cell invasion. "P<0.05. siRNA, small interfering RNA; Glut1, glucose receptor 1 .

Recently, Liao et al (22) evaluated the blood glucose level of 2,048 patients with pancreatic cancer. A dose-response meta-analysis demonstrated that every $0.56 \mathrm{mmol} / \mathrm{l}$ increase in FBG was associated with a $14 \%$ increase in the rate of 

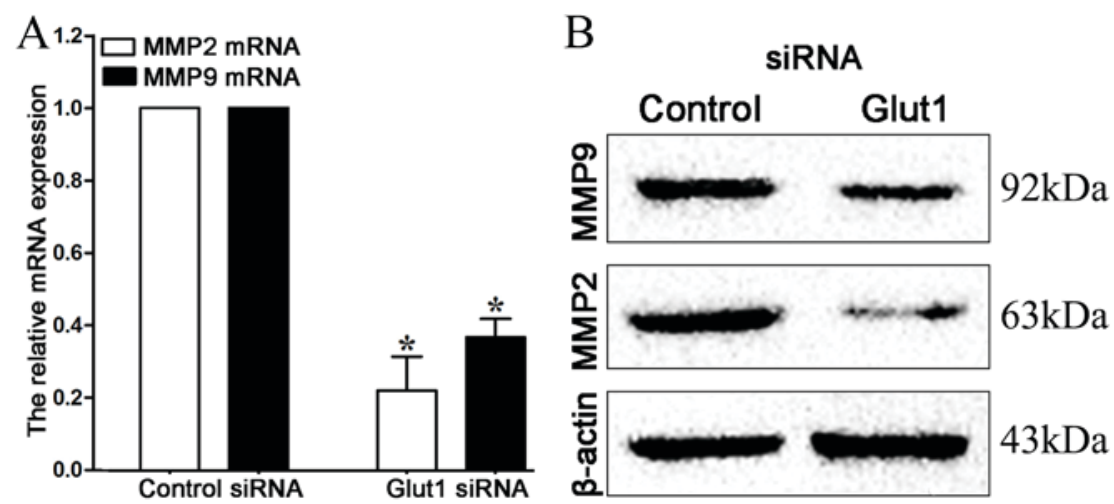

Figure 4. (A) Downregulation of Glut1 inhibited MMP2 and MMP9 mRNA expression in MCF-7 cells. (B) Downregulation of Glut1 inhibited MMP2 and MMP9 protein expression in MCF-7 cells. "P<0.05. Glut1, glucose receptor 1; MMP2, matrix metalloproteinase 2; MMP9, matrix metalloproteinase 9; siRNA, small interfering RNA.

pancreatic cancer. Hosokawa et al (23) reviewed the prognosis of 344 patients with hepatocellular carcinoma (HCC) subsequent to radiofrequency ablation (RFA), and found the status of hyperglycemia is positively correlated with cancer recurrence. The 3-year recurrent rate of HCC with T2DM subsequent to RFA treatment was $93.8 \%$, while only $73.0 \%$ for HCC patients without T2DM. In the present study, it was similarly found that IDBC patients with T2DM often had a larger tumor size, and increased metastasis. Therefore, T2DM may promote tumor growth and invasion in breast cancer. Additionally, it was found that, compared with non-metastatic IDBC patients, the serum FBG level was higher in metastatic IDBC patients. Additional in vitro assays also confirmed that high glucose levels in the tumor microenvironment may enhance MCF-7 cell invasion. Consequently, as a systematic disease, T2DM can affect the glucose concentration of the tumor microenvironment, which eventually changes cell biological behaviors.

Overexpression of Glut1 has been confirmed in numerous types of cancer (24); however, the function of Glut1 in IDBC patients combined with T2DM remains unknown. The extracellular matrix can be destroyed by matrix metalloproteinases, which is the key process of cancer metastasis (25). In pancreatic cancer, Glut1 may promote the expression of MMP2 and enhance MIAPaCa-2 and PANC-1 cell invasion (26). $\mathrm{Xu}$ et al (27) reported that the downregulation of Glut1 by antisense oligodeoxynucleotide significantly inhibits MMP2 expression in laryngeal carcinoma Hep-2 cells. To investigate the functions and mechanisms of Glut1 in IDBC, the present study first tested the mRNA and protein levels of Glut1, MMP2 and MMP9 in IDBC tissues, and demonstrated that the expression of these 3 factors is higher in IDBC tissues with T2DM. Suppressing Glut1 expression in MCF-7 cells cultured in high glucose medium markedly reduced the number of invaded cells, this may be the result of lower expression of MMP2 and MMP9, which were confirmed by RT-qPCR and western blot analysis.

In conclusion, IDBC patients with T2DM experienced an increased amount of malignant clinicopathological features in comparison to patients with IDBC alone. IDBC cells cultured in a high glucose microenvironment are more invasive, and this may be a result of the excessive activation of the Glut1/MMP2/MMP9 axis. Downregulation of Glut1 may suppress IDBC progression by impairing cell invasion for T2DM patients.

\section{Acknowledgements}

The present study was supported by a grant from the Key Science and Technology Project of Science and Technology Department of Henan Province (grant no. 122102310535).

\section{References}

1. Ozyalvacli G, Yesil C, Kargi E, Kizildag B, Kilitci A and Yilmaz F: Diagnostic and prognostic importance of the neutrophil lymphocyte ratio in breast cancer. Asian Pac J Cancer Prev 15: 10363-10366, 2014.

2. Joung KH, Jeong JW and Ku BJ: The association between type 2 diabetes mellitus and women cancer: The epidemiological evidences and putative mechanisms. Biomed Res Int 2015: 920618, 2015.

3. Nie SP, Chen H, Zhuang MQ and Lu M: Anti-diabetic medications do not influence risk of lung cancer in patients with diabetes mellitus: A systematic review and meta-analysis. Asian Pac J Cancer Prev 15: 6863-6869, 2014.

4. Wintrob ZA, Hammel JP, Khoury T, Nimako GK, Fu HW, Fayazi ZS, Gaile DP, Forrest A and Ceacareanu AC: Insulin use, adipokine profiles and breast cancer prognosis. Cytokine 89: 45-61, 2017.

5. Ma FJ, Liu ZB, Qu L, Hao S, Liu GY, Wu J and Shao ZM: Impact of type 2 diabetes mellitus on the prognosis of early stage triple-negative breast cancer in People's Republic of China. OncoTargets Ther 7: 2147-2154, 2014.

6. Chen Z, Meng Z, Jia L and Cui R: The tumor microenvironment and cancer. Biomed Res Int 2014: 573947, 2014.

7. Adham SA, Al Rawahi H, Habib S, Al Moundhri MS, Viloria-Petit A and Coomber BL: Modeling of hypo/hyperglycemia and their impact on breast cancer progression related molecules. PLoS One 9: e113103, 2014.

8. Yang W and Lu Z: Nuclear PKM2 regulates the Warburg effect. Cell Cycle 12: 3154-3158, 2013.

9. Xu Q, Liu X, Zheng X, Yao Y and Liu Q: PKM2 regulates Gli1 expression in hepatocellular carcinoma. Oncol Lett 8: 1973-1979, 2014.

10. Govers R: Cellular regulation of glucose uptake by glucose transporter GLUT4. Adv Clin Chem 66: 173-240, 2014.

11. Tang Z, Wang J, Zhang H, Sun L, Tang F, Deng Q and Yu J: Associations between diabetes and quality of life among breast cancer survivors. PLoS One 11: e0157791, 2016.

12. Heilig CW, Deb DK, Abdul A, Riaz H, James LR, Salameh J and Nahman NS Jr: GLUT1 regulation of the pro-sclerotic mediators of diabetic nephropathy. Am J Nephrol 38: 39-49, 2013.

13. McKinnon B, Bertschi D, Wotzkow C, Bersinger NA, Evers J and Mueller MD: Glucose transporter expression in eutopic endometrial tissue and ectopic endometriotic lesions. J Mol Endocrinol 52: 169-179, 2014. 
14. Osugi J, Yamaura T, Muto S, Okabe N, Matsumura Y, Hoshino M, Higuchi M, Suzuki H and Gotoh M: Prognostic impact of the combination of glucose transporter 1 and ATP citrate lyase in node-negative patients with non-small lung cancer. Lung cancer 88: 310-318, 2015.

15. Nam SO, Yotsumoto F, Miyata K, Fukagawa S, Yamada H, Kuroki M and Miyamoto S: Warburg effect regulated by amphiregulin in the development of colorectal cancer. Cancer Med 4 575-587, 2015

16. Zhang J, Tu K, Yang W, Li C, Yao Y, Zheng X and Liu Q: Evaluation of Jagged 2 and Gli1 expression and their correlation with prognosis in human hepatocellular carcinoma. Mol Med Rep 10: 749-754, 2014.

17. Livak KJ and Schmittgen TD: Analysis of relative gene expression data using real-time quantitative PCR and the 2(-Delta Delta C(T)) Method. Methods 25: 402-408, 2001.

18. Liao H, Wang Z, Deng Z, Ren H and Li X: Curcumin inhibits lung cancer invasion and metastasis by attenuating GLUT1/MT1-MMP/MMP2 pathway. Int J Clin Exp Med 8: 8948-8957, 2015.

19. Zeng H, Zheng R, Guo Y, Zhang S, Zou X, Wang N, Zhang L, Tang J, Chen J, Wei K, et al: Cancer survival in China, 2003-2005: A population-based study. Int J Cancer 136: 1921-1930, 2015.

20. Janssen S, Holz-Sapra E, Rades D, Moser A and Studer G: Nipple-sparing mastectomy in breast cancer patients: The role of adjuvant radiotherapy (Review). Oncol Lett 9: 2435-2441, 2015.
21. Liu X, Hemminki K, Forsti A, Sundquist K, Sundquist J and Ji J: Cancer risk in patients with type 2 diabetes mellitus and their relatives. Int J Cancer 137: 903-910, 2015.

22. Liao WC, Tu YK, Wu MS, Lin JT, Wang HP and Chien KL: Blood glucose concentration and risk of pancreatic cancer: Systematic review and dose-response meta-analysis. BMJ 349: g7371, 2015.

23. Hosokawa T, Kurosaki M, Tsuchiya K, Matsuda S, Muraoka M, Suzuki Y, Tamaki N, Yasui Y, Nakata T, Nishimura T, et al: Hyperglycemia is a significant prognostic factor of hepatocellular carcinoma after curative therapy. World J Gastroenterol 19: 249-257, 2013

24. Labak CM, Wang PY, Arora R, Guda MR, Asuthkar S, Tsung AJ and Velpula KK: Glucose transport: Meeting the metabolic demands of cancer, and applications in glioblastoma treatment. Am J Cancer Res 6: 1599-1608, 2016.

25. Pal S, Moulik S, Dutta A and Chatterjee A: Extracellular matrix protein laminin induces matrix metalloproteinase-9 in human breast cancer cell line mcf-7. Cancer Microenviron 7: 71-78, 2014.

26. Ito H, Duxbury M, Zinner MJ, Ashley SW and Whang EE: Glucose transporter-1 gene expression is associated with pancreatic cancer invasiveness and MMP-2 activity. Surgery 136: 548-556, 2004.

27. Xu YY, Bao YY, Zhou SH and Fan J: Effect on the expression of MMP-2, MT-MMP in laryngeal carcinoma Hep-2 cell line by antisense glucose transporter-1. Arch Med Res 43: 395-401, 2012. 